1

\title{
Roles of Intramolecular and Intermolecular Interactions in Functional Regulation of the Hsp70 J-protein Co-chaperone Sis1
}

\author{
Hyun Young Yu ${ }^{1}$, Thomas Ziegelhoffer ${ }^{1}$, Jerzy Osipiuk ${ }^{2}$, Szymon J. Ciesielski ${ }^{1}$, Maciej \\ Baranowski $^{3}$, Min Zhou ${ }^{2}$, Andrzej Joachimiak ${ }^{2,4}$ and Elizabeth A. Craig ${ }^{1 *}$
}

\footnotetext{
${ }^{1}$ Department of Biochemistry, University of Wisconsin-Madison, 433 Babcock Drive, Madison, WI 53706, USA

${ }^{2}$ Midwest Center for Structural Genomics, Department of Biosciences, Argonne National Laboratory, 9700 South Cass Avenue, Building 202, Argonne, IL 60439, USA

${ }^{3}$ Laboratory of Biopolymer Structure, Intercollegiate Faculty of Biotechnology, University of Gdansk and Medical University of Gdansk, Gdansk 80822, Poland

${ }^{4}$ Department of Biochemistry and Molecular Biology, University of Chicago, 920 East 58th Street, Chicago Il 60637, USA
}

*corresponding author: ecraig@wisc.edu; phone: 1-608 263 7105; FAX: 1-608 2623453 


\section{Abstract}

Unlike other Hsp70 molecular chaperones, those of the eukaryotic cytosol have four residues, EEVD, at their C-termini. EEVD(Hsp70) binds adaptor proteins of the Hsp90 chaperone system and mitochondrial membrane preprotein receptors, thereby facilitating processing of Hsp70bound clients through protein folding and translocation pathways. Among J-protein cochaperones functioning in these pathways Sis1 is unique, as it also binds the $\operatorname{EEVD}(\mathrm{Hsp} 70)$ motif. However, little is known about the role of the Sis1:EEVD(Hsp70) interaction. We found that deletion of $\operatorname{EEVD}(\mathrm{Hsp} 70)$ abolished the ability of Sis1, but not the ubiquitous J-protein Ydj1, to partner with Hsp70 in in vitro protein refolding. Sis1 co-chaperone activity with Hsp $70_{\triangle E E V D}$ was restored upon substitution of a glutamic acid of the J-domain. Structural analysis revealed that this key glutamic acid, which is not present in Ydj1, forms a salt bridge with an arginine of the immediately adjacent glycine-rich region. Thus, restoration of Sis1 in vitro activity suggests that intramolecular interaction(s) between the J-domain and glycine-rich region controls co-chaperone activity, which is optimal only when Sis1 interacts with the EEVD(Hsp70) motif. Yet, we found that disruption of the Sis1:EEVD(Hsp70) interaction enhances the ability of Sis1 to substitute for Ydj1 in vivo. Our results are consistent with the idea that interaction of Sis1 with $\operatorname{EEVD}(\mathrm{Hsp} 70)$ minimizes transfer of Sis1-bound clients to Hsp70s that are primed for client transfer to folding and translocation pathways by their preassociation with EEVD-binding adaptor proteins. These interactions may be one means by which cells triage Ydj1- and Sis1-bound clients to productive and quality control pathways, respectively. 


\section{Introduction}

By interacting with a wide variety of client proteins, molecular chaperones function in many cellular processes, ranging from the folding of nascent polypeptides to the remodeling of multimeric protein complexes [1, 2]. Ubiquitous Hsp70 systems are central players in cellular chaperone networks, performing a particularly important role in protein folding and translocation, as well as in prioritizing proteins for folding pathways and the quality control systems of protein aggregation and proteolysis [3]. All Hsp70s, regardless of the cellular compartment in which they reside, have a C-terminal peptide binding domain, which interacts with short segments of client proteins, and an N-terminal adenine nucleotide-binding domain, which regulates client interaction [4]. Obligatory co-chaperones, called J-proteins, interact at the domain interface, stimulating ATP hydrolysis, thereby stabilizing Hsp70's interaction with client [5]. This role is carried out by the highly conserved helical J-domain found in all J-proteins. Some J-proteins also bind client proteins, serving to target them for Hsp70 binding.

Unlike other Hsp70s, eukaryotic cytosolic Hsp70s (called Ssa in Saccharomyces cerevisiae) also have a conserved EEVD tetrapeptide at their extreme C-terminus [6]. This EEVD motif, called EEVD(Hsp70) throughout, interacts with targeting/adaptor factors that facilitate trafficking of associated client proteins along Hsp90 folding pathways and assists targeting clients to mitochondria [7-11]. Sis1 co-chaperone, one of the two most abundant soluble cytosolic J proteins, also binds EEVD(Hsp70); in contrast, the other, Ydj1 does not [12, 13]. Although Ydj1 has a Zn binding domain, but Sis1 does not, the two proteins have many similarities [5]. Both have an N-terminal J-domain, followed by a glycine-rich region, two structurally similar domains with ß-barrel topology (CTD1 and CTD2), which contain the clientbinding cleft of both proteins and the $\operatorname{EEVD}(\mathrm{Hsp} 70)$ interaction site of Sis1, and a C-terminal 
dimerization domain. Sis1 and Ydj1, play partially overlapping roles in determining the fate of polypeptide chains, consistent with their distinct, yet overlapping, specificity for client binding [14-16]. Ydj1 plays well-established roles in driving client proteins into Hsp90 folding pathways and across cellular membranes into organelles. On the other hand, recent data indicate that Sis1 is important in protein quality control, targeting clients to centers of aggregation and proteolysis [17-19].

As the functional relevance of the $\operatorname{Sis} 1: \operatorname{EEVD}(\operatorname{Hsp} 70)$ interaction is unknown, we analyzed the consequences of disrupting it. On one hand, we found the Sis1:EEVD(Hsp70) interaction to be critical for Sis1 in Hsp70-mediated protein refolding in vitro. However, this requirement is relieved by alteration of a single residue in the J-domain, which was shown to interact with the glycine-rich region. These results suggest that Sis1 partnership with Hsp70 is optimized through intramolecular and intermolecular interactions. On the other hand, we found that disruption of the Sis1:EEVD(Hsp70) interaction enhanced the ability of Sis1 to substitute for Ydj1 in vivo. Our results suggest that interaction of Sis1 with $\operatorname{EEVD}(\mathrm{Hsp} 70)$ may diminish the chances of Sis1-bound clients being transferred to folding and translocation pathways, providing fine-tuning of client targeting between folding and quality control pathways.

\section{Results}

\section{EEVD(Hsp70) motif is required for refolding activity of Sis1, but not Ydj1 and Xdj1}

As a first step in our analysis of the importance of the interaction of Sis1 with EEVD(Hsp70), we compared the ability of the full-length Hsp70 Ssa1 and Ssa1 lacking the C-terminal four residues (called Hsp70 and Hsp70 ${ }_{\triangle \mathrm{EEVD}}$, respectively, throughout) to function with Sis1 and Ydj1 as Jprotein partners in facilitating protein refolding. Sis1 and Ydj1 were similarly efficient in 
refolding of urea-denatured luciferase when partnering with full-length Hsp70. However, while Ydj1 facilitated refolding nearly as efficiently when partnering with $\mathrm{Hsp} 70_{\triangle \mathrm{EEVD}}$ as with fulllength Hsp70, Sis1 did not promote refolding with $\mathrm{Hsp}_{40_{\triangle E E V D}}$ (Fig. 1a). Within 45 min 100\% of

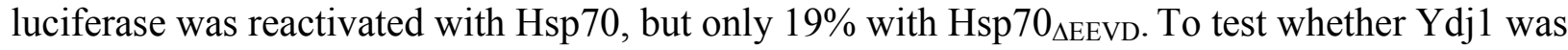
unique in being able to function efficiently with Hsp $70_{\triangle \mathrm{EEVD}}$ we also tested Xdj1, a paralog of Ydj1 arising from a gene duplication during the fungal lineage [20]. Xdj1 was also able to promote luciferase refolding with $\mathrm{Hsp} 70_{\triangle \mathrm{EEVD}}$.

The luciferase refolding reactions discussed above included Hsp104, a chaperone specialized in resolubilization of aggregates, as well as the nucleotide exchange factor Sse1. To ascertain whether these two components played a major role in the difference between Sis1 and Ydj1/Xdj1-driven refolding by Hsp70 and Hsp70 2 EEVD, we carried out refolding assays in which luciferase was partially denatured by guanidinium hydrochloride. This is a milder treatment than the urea denaturation method, and, as expected [21], refolding occurred when only Hsp70 and Jprotein were included in the reaction (Fig 1b). As with the assay containing Hsp104 and Sse1, Sis 1 did not partner with Hsp $70_{\triangle \mathrm{EEVD}}$. We conclude that, independent of other chaperone proteins, the EEVD motif of Hsp70 plays an important role in partnering with Sis1, but not with Ydj1 or Xdj1, in protein refolding.

To determine whether this difference between Sis1 and Ydj1/Xdj1 in ability to partner with Hsp70 and $\mathrm{Hsp} 70_{\triangle \mathrm{EEVD}}$ extended to other clients, we tested refolding of malate dehydrogenase (MDH) after denaturation by heat treatment. All three J-proteins partnered with full-length Hsp70, reaching nearly $100 \%$ refolding within 90 min (Fig. 1c; Supplementary Fig. 1a). However, when Sis 1 was paired with $\mathrm{Hsp} 70_{\triangle \mathrm{EEVD}}, \mathrm{MDH}$ activity did not rise above background levels. On the other hand, in reactions combining Xdj1 and Ydj1 with Hsp $70_{\Delta E E V D,}$ 
MDH activity reached 88 and $72 \%$, respectively within that time. Our results support the idea that interaction between Sis 1 and $\operatorname{EEVD}(\mathrm{Hsp} 70)$ is important for Sis1's ability to function efficiently in protein refolding in vitro.

\section{Substitution of J-domain of Ydj1 or Xdj1 overcomes Sis1's dependence on (EEVD)Hsp70} To better understand the basis of the requirement of $\operatorname{EEVD}(\mathrm{Hsp} 70)$ in the function of Sis1, but not Ydj1/Xdj1, we constructed chimeras. We swapped Ydj1's and Xdj1's J-domains for that of Sis 1, thereby generating $\mathrm{J}_{\mathrm{Ydj} 1} \operatorname{Sis} 1$ and $\mathrm{J}_{\mathrm{Xdj} 1} \operatorname{Sis} 1$. Surprisingly, both $\mathrm{J}_{\mathrm{Ydj} 1} \operatorname{Sis} 1$ and $\mathrm{J}_{\mathrm{Xdj} 1} \mathrm{Sis} 1$ partnered with $\mathrm{Hsp} 70_{\triangle \mathrm{EEVD}}$ substantially better than Sis1 in refolding of luciferase and $\mathrm{MDH}$ (Fig. 2a,b; Supplementary Fig. 1b). For example, in reactions containing $\mathrm{J}_{\mathrm{Ydj} 1} \mathrm{Sis} 1$ and $\mathrm{J}_{\mathrm{Xdj}} \mathrm{Sis} 1$, 100 and $85 \%$ of luciferase activity, respectively, was recovered, compared to only $19 \%$ for wild type Sis1 within 60 min.

We next compared the ATPase stimulatory ability of Sis $1, \mathrm{Ydj} 1$ and the chimeras using a preformed ${ }^{32} \mathrm{P}$-ATP-Hsp70 complex (Fig. 2c). Ydj1 stimulated the ATPase activity of Hsp70 more effectively than Sis1, resulting in $95 \%$ hydrolysis within two minutes. $78 \%$ of the ATP was hydrolyzed by 12 min in reactions containing Sis 1 , at which point $41 \%$ hydrolysis had occurred in the control reaction having no J-protein. Both the $\mathrm{J}_{\mathrm{Ydj} 1} \mathrm{Sis} 1$ and $\mathrm{J}_{\mathrm{Xdj} 1} \mathrm{Sis} 1$ chimera stimulated ATP hydrolysis more effectively than wild type Sis1, with 74 and $63 \%$ hydrolysis achieved within two minutes. Thus swapping of Ydj1's or Xdj1's J-domain for that of Sis1 both overcame Sis 1's dependence on the EEVD motif of Hsp70 for refolding and increased Sis1's ability to stimulate Hsp70's ATPase activity, suggesting a functional difference between these J-domains.

\section{Alteration of Sis1 J-domain overcomes dependence on EEVD(Hsp70) for in vitro refolding}


To find clues as to what residues might be responsible for these functional differences between Jdomains, we compared their sequences. We found three regions (labeled A, B and C in Fig. 3a) having substantial sequence differences. Two of these, segments A and B, mainly encompass loops between helices; the majority of residues of the third, segment $\mathrm{C}$, is mainly part of helix 3 . Constructs were made such that A, B and C segments of Ydj1 (residues 14-20, 38-44 and 52-61, respectively) were substituted individually for those of Sis1. The activities of the resulting variants were then tested. The two constructs having exchanged segments of loops, $\mathrm{J}_{\mathrm{Ydj1} 1 \mathrm{~A}} \mathrm{Sis} 1$ and $\mathrm{J}_{\mathrm{Ydj} 1-\mathrm{B}} \mathrm{Sis} 1$ were, like Sis1, unable to partner with $\mathrm{Hsp} 70_{\triangle \mathrm{EEVD}}$ (Fig. 3b Supplementary Fig. 1c). On the other hand, $\mathrm{J}_{\mathrm{Ydj1} 1 \mathrm{C}} \mathrm{Sis} 1$ containing the 52-61 segment of $\mathrm{Ydj} 1$ functioned in reactivation of luciferase and $\mathrm{MDH}$ with $\mathrm{Hsp} 70_{\triangle \mathrm{EEVD}}$, as well as it did with wild type Hsp70. Thus, we hypothesized that helix 3 is responsible for the functional difference between the Sis 1 and Ydj1/Xdj1 J-domains.

The sequence of Ydj1 and Xdj1 are identical over the 10 residue $\mathrm{C}$ segment. However, four differ in Sis1 (E50, F52, N56 and Q59). Thus, we substituted the Ydj1/Xdj1 residues individually into Sis1's J-domain and tested the variants for refolding activity when partnering with Hsp $70_{\triangle \mathrm{EEVD}}$. While all four variants were active with full-length Hsp70, three (Sis $1_{\mathrm{F} 52 \mathrm{Y}}$, Sis $1_{\mathrm{N} 56 \mathrm{~S}}$ and $\left.\mathrm{Sis} 1_{\mathrm{Q} 59 \mathrm{E}}\right)$ behaved similarly to wild type $\mathrm{Sis} 1$, that is, they were inactive with Hsp $70_{\triangle E E V D}$. However, nearly $90 \%$ of the denatured luciferase was refolded within 60 min in reactions containing Sis $1_{\mathrm{E} 50 \mathrm{~A}}$ and $\mathrm{Hsp} 70_{\triangle \mathrm{EEVD}}$ (Fig. 4a). Similar results were obtained in MDH refolding assays. The activity of Sis $1_{\mathrm{E} 50 \mathrm{~A}}$ with $\mathrm{Hsp} 70_{\triangle \mathrm{EEVD}}$ was indistinguishable from that with full-length Hsp70, while the activities of the other three variants were substantially less (Fig 4b, Supplementary Fig. 1d). Thus, alteration of a single residue, E50, in Sis1's J-domain 
compensates for the deficiency in Sis1's protein refolding activity caused by the absence of the Hsp70 EEVD motif.

To better understand the relationship between alteration of E50 alteration and activity of the Sis1 J-domain we tested the ability of two additional constructs to stimulate the ATPase activity of Hsp70: full-length Sis1 with the E50A substitution in its J-domain (Sis $\left.1_{\mathrm{E} 50 \mathrm{~A}}\right)$ and a chimera substituting the wild type Sis1 J-domain for that of Ydj1 ( $\left.\mathrm{J}_{\text {Sis1 }} \mathrm{Ydj1}\right)$. The E50A alteration enhanced Sis1's ability to stimulate Ssa1's ATPase activity (Fig. 4c). This enhancement was similar to that resulting from substitution of the entire J-domain of Ydj1 (i.e. $\mathrm{J}_{\mathrm{Sis} 1} \mathrm{Ydj} 1$ ), suggesting that the $\mathrm{J}$-domain of Sis 1 is not inherently less efficient than the $\mathrm{J}$-domain of Ydj1.

\section{Interaction of Sis1 E50 with R73 of glycine-rich region}

To understand how E50 might affect Sis 1 function we carried out structural analysis of the Jdomain of S. cerevisiae Sis1 using X-ray crystallography. To increase the odds of crystallization, we tested several $\mathrm{N}$-terminal fragments of Sis1, varying in length from 74 to 125 residues. We obtained several protein crystal forms for the 74 - and 89 -residue fragments. The best quality Xray diffraction was collected for the 89-residue $\mathrm{N}$-terminal domain construct. We obtained atomic resolution diffraction data $(1.25 \AA)$ with $60 \%$ reflection completeness in the highest resolution shell due to the crystal anisotropicity and $90 \%$ completeness at $1.37 \AA$ resolution (Supplementary Table 1,2). The final model consists of Sis1 residues 1-81, which includes the key $\alpha$-helix containing E50, as well as a portion of the glycine-rich region. It lacks the $8 \mathrm{C}$ terminal residues, which were not defined in electron density maps, as well as the three $\mathrm{N}$ terminal residues remaining after cleavage of the purified protein fusion with TEV protease. 
The overall structure of the 81-residue N-terminal Sis 1 fragment is comprised of five $\alpha$ helices (Fig. 5a). The first four helices form a typical J-domain structure with the conserved HPD motif residing between helices $\mathrm{H} 2$ and $\mathrm{H} 3$ [22]. The same structural futures have been observed in X-ray structures of other J domains, the closest being the Sis1 homolog from Caenorhabditis elegans, Dnj 12J (PDB ID: 2OCH), as well as in NMR structures of other J-domains (e.g. PDB ID: 1BQZ and 2DN9). The fifth helix, residues $68-74$, is part of the glycine-rich region. The unique feature of the structure is the presence of a salt bridge between the side chains of E50 of $\alpha$-helix 3 of the J-domain and R73 of $\alpha$-helix 5 of the glycine-rich region (Fig. 5a). The residues are very well defined in the structure with side-chains B-factors in the 14-19 $\AA^{2}$ range and outstanding electron density maps. The E50/R73 contacts are the only hydrogen bonds between the J-domain and glycine-rich region in the structure and undoubtedly stabilize interaction of the J-domain with glycine-rich region.

We reasoned that if the salt-bridge observed between E50 and R73 revealed by our structural analysis is functionally relevant, alteration of R73 would be expected to affect Sis1 function similarly to that caused by alteration of E50. Therefore, we purified Sis $1_{\mathrm{R} 73 \mathrm{~A}}$ and tested its activity in in vitro refolding assays. We found that alteration of R73 to A partially restored the ability of Sis1 to function in refolding of luciferase and MDH with $\mathrm{Hsp} 70_{\triangle \mathrm{EEVD}}$ (Fig. 5b,c; Supplementary Fig 1e) consistent with functional importance of the E50:R73 salt bridge.

\footnotetext{
Sis1 variants defective in EEVD(Hsp70) interaction have reduced in vitro folding activity

The experiments described above underline the importance of EEVD(Hsp70) for functional interaction with Sis1, but do not establish that it is the physical interaction between the two proteins per se that is important. To address this question, we purified Sis1 variants having
} 
alterations of CTD1 residues demonstrated to directly interact with the EEVD of the Hsp70 Ssa1 [12]. We then assessed both binding to Hsp70 and activity in in vitro refolding assays. Three residues were changed: Sis1 residue K199, which interacts with Ssa1 residue D642, as well as Sis 1 residues K202 and K214, both of which interact with E640 of Ssa1 Hsp70 (Fig. 6a). To compare the interaction of wild type and variant Sis1 proteins with Hsp70, we used an ELISA assay in which the amount of Sis1 bound to Hsp70 immobilized in wells was measured using Sis 1-specific antibodies. At a concentration of wild type Sis1 of $200 \mathrm{nM}$, binding to Hsp70 approached saturation, with 50\% maximal binding achieved at approximately $30 \mathrm{nM}$. The variant

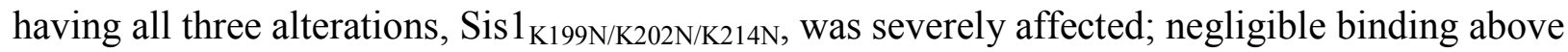
background levels was detected at the highest concentration tested, $200 \mathrm{nM}$. Binding of each single alteration variant was detected, but all were significantly defective. The ability of variants to refold luciferase and MDH followed a similar pattern. The activity of $\mathrm{Sis} 1_{\mathrm{K} 199 \mathrm{~N} / \mathrm{K} 202 \mathrm{~N} / \mathrm{K} 214 \mathrm{~N}}$ was negligible in both the luciferase and MDH refolding assays (Fig 6b; Supplementary Fig. 1f).

As described above (Fig. 4), the E50A substitution overcame Sis1's defect in refolding by $\mathrm{Hsp} 70_{\triangle \mathrm{EEVD}}$. As a test of whether the lack of refolding function of $\mathrm{Sis} 1_{\mathrm{K} 199 \mathrm{~N} / \mathrm{K} 202 \mathrm{~N} / \mathrm{K} 214 \mathrm{~N} \text { was }}$ also overcome by the E50A alteration we combined the four alterations, generating

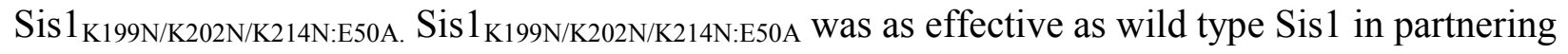
with Hsp70 in refolding of both luciferase and MDH (Fig. 6c; Supplementary Fig. 1g), supporting the idea that the functional defect of $\operatorname{Sis} 1_{\mathrm{K} 199 \mathrm{~N} / \mathrm{K} 202 \mathrm{~N} / \mathrm{K} 214 \mathrm{~N}}$ is due to its lack of interaction with Hsp70 via its EEVD motif. We also tested the effect of altering R73, which

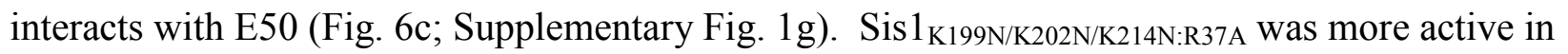

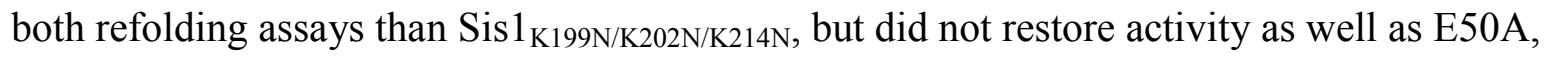


similar to what was seen for the ability of the two alterations to overcome the folding defect of Hsp70 $0_{\triangle E E V D}$ (Fig. 5b, c).

\section{Disruption of Sis1:EEVD(Hsp70) interaction enhances Sis1's ability to substitute for Ydj1}

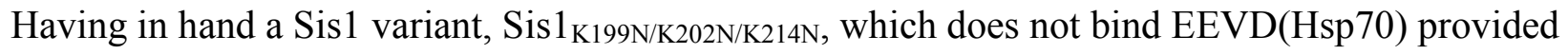
us the opportunity to assess in vivo effects of the lack of interaction between $\operatorname{EEVD}(\operatorname{Hsp} 70)$ and Sis 1 . We tested the effect of the $\operatorname{sis}_{\text {K199N/K202N/K214N }}$ mutation in two genetic backgrounds: 1) in an otherwise wild type background, and 2) in the presence of a partial loss of function YDJ1 allele, $y d j 1_{1-134}$. The first test was to determine if loss of $\operatorname{EEVD}(\mathrm{Hsp} 70)$ interaction substantially affected Sis1's ability to carry out its housekeeping functions. We found, no obvious growth defect of $\operatorname{sis} 1_{K 199 N / K 202 N / K 214 \mathrm{~N}}$ at a variety of temperatures and culture conditions (Fig. 7a) even though Sis1 is essential [23].

The second test was set up to determine if interaction of Sis1 with EEVD(Hsp70) might impede its ability to substitute for Ydj1 in vivo. We reasoned that an Hsp70 whose EEVD is interacting with a receptor/adaptor might be less likely to partner with Sis1 than one whose EEVD is free to interact with Sis1. Because $\Delta y d j 1$ cells grow so poorly, making genetic manipulation difficult, we used cells expressing Ydj1 1-134. The presence of Ydj1's J-domain and glycine-rich region partially overcome the very severe growth defect of $\Delta y d j 1$. Cells expressing Ydj $1_{1-134}$ along with wt Sis 1 are temperature sensitive for growth [14], failing to form colonies at $34^{\circ} \mathrm{C}$ (Fig 7). However, $y d j 1_{1-134}$ cells expressing Sis $1_{\mathrm{K} 199 \mathrm{~N} / \mathrm{K} 202 \mathrm{~N} / \mathrm{K} 214 \mathrm{~N}}$ supported robust growth at $34^{\circ} \mathrm{C}$ (Fig. 7b), even though the levels of Sis1 protein were very similar (Fig. 7d).

The observed suppression is consistent with the idea that elimination of the Sis1:EEVD(Hsp70) interaction allows Sis1 to more effectively partner with an Hsp70 that has a EEVD binding receptor bound (Fig. 8), rather than selectively interact with Hsp70s having their 
EEVD free. If, indeed, the elimination of the $\operatorname{EEVD}(\operatorname{Hsp} 70)$ binding site on Sis1 is the cause of the suppression then the suppression would be expected to occur whether or not wild type Sis1 was present, that is the mutation results in a "gain of function". To test this idea, we expressed wt Sis 1 and Sis $1_{\mathrm{K} 199 \mathrm{~N} / \mathrm{K} 202 \mathrm{~N} / \mathrm{K} 214 \mathrm{~N}}$ simultaneously in the test strain expressing $\mathrm{Ydj} 1_{1-134}$. We found that suppression of the temperature-sensitive growth defect of $y d j 1_{1-134}$ cells was similar in the

presence or absence of wild type Sis1 (Fig. 7b,c). Thus, the absence of EEVD(Hsp70) binding by Sis 1 results in partial suppression of the growth defects associated with partial loss of function of Ydj1, whether or not wild type Sis1 is present.

\section{Discussion}

Several results reported here point to the conclusion that the interaction of the Sis1 co-chaperone with the EEVD motif at Hsp70's C-terminus is important for the cooperation of these two

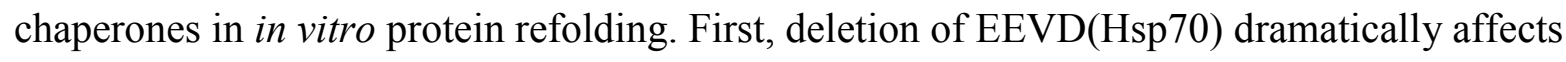
Hsp70's ability to partner with Sis1, but not with two other J-proteins, Ydj1 and Xdj1, which do not interact with EEVD(Hsp70). Second, alteration of the EEVD binding site of Sis1 also dramatically reduced the refolding activity of Sis1, even with full-length Hsp70. Third, substitution of a single residue, E50, of the Sis1 J-domain substantially overcame the folding deficiency caused by the absence of the EEVD of Hsp70, as well as a defective EEVDinteraction site on Sis1. This result is particularly informative because it addresses potential concerns that modification of the EEVD binding site of Sis1 affects other important activities (e.g. client binding). The fact that a single residue can be substituted and suppress activities of both the Hsp70 and Sis1 variants argues strongly for an important role of the Sis1:EEVD(Hsp70) interaction. 
The fact that substitution of either interacting residues, that is E50 or R73, has similar effects strongly points to the importance of this salt bridge between the J-domain and the glycine-rich region. That either exchanging the Sis1 J-domain for that of Ydj1 or altering E50 increases the ATPase stimulatory ability of the full-length protein suggests that the salt-bridge reduces J-domain activity. Perhaps this E50:R73 interaction decreases the flexibility of the Jdomain, constraining its activity. What might be the consequence of the Sis 1 interaction with the EEVD motif of Hsp70? One possibility is a role in positioning Hsp70's ATPase domain and the $\mathrm{J}$-domain in a productive conformation. It is also possible that a lower stimulatory capacity is sufficient for Sis1 function, because the Sis1:EEVD(Hsp70) interaction itself serves to increase the local concentration of the J-domain. Further structural studies will be needed to answer such questions.

At first glance it seems surprising that we found no obvious growth defect caused by a defective Sis1:EEVD(Hsp70) interaction in otherwise wild type cells, as SIS1 is an essential gene [23]. However, it has been known for some time that yeast cells grow quite vigorously, at least under standard laboratory conditions, even when Sis1 is expressed at a substantially lower level than normal [24]. In addition, a fragment containing only the J-domain and glycine-rich region is sufficient to carry out the essential functions of Sis1, as it supports viability [25, 26]. We favor the idea that protein folding per se is not an essential function of Sis1 under normal conditions when full-length Ydj1, which is normally more abundant than Sis1 [27], is present. Indeed, we reported previously that CTD1/CTD2 of either Sis1 or Ydj1, but not both, was required for cell viability [14]. However, as the glycine-rich region of Sis1, but not Ydj1, is specifically required for cell viability, we think that this region carries out critical roles, perhaps in regulating Jdomain function. A part of that regulation is likely due to the interactions between the J-domain 
and the glycine-rich region as illustrated by our finding, described here, of the E50:R73 salt bridge.

Our finding that disruption of the $\operatorname{EEVD(Hsp70):Sis1~interaction~enhances~Sis1's~ability~to~}$ compensate for diminished Ydj1 activity is consistent with the idea that a role of this interaction is to divert Sis1-bound client proteins away from entering the "productive" pathways of Hsp90 driven protein folding and import into mitochondria (Fig. 8). This interpretation is consistent with recent results [17-19] indicating that Sis1 may be more involved in triaging its clients to quality control centers, that is aggregation and proteolytic pathways, than in protein folding in vivo. Targeting proteins for centers of aggregation and proteolysis, such as Btn2 and Cur1 [28, 29], which interact with Sis1, but not Ydj1, have been identified. Such mechanisms that act to target Sis 1 and its clients to such centers, coupled with the diversion away from productive pathways via the Sis1:EEVD(Hsp70) interaction discussed here, are complementary in promoting protein homeostasis. Interestingly, as the human Sis1 ortholog also interacts with EEVD(Hsp70) [30] such mechanisms may well be conserved.

\section{Materials and Methods}

\section{Protein purification, plasmids and yeast strains}

Ssa1 was purified from yeast using Ni chromatography, as described in Pfund et al [31]. SIS1, $Y D J 1$ and $X D J 1$ were cloned into pMAL-His-TEV [32], and expressed in Rosetta 2 (DE3) pLys E. coli cells. Cells were grown at $37^{\circ} \mathrm{C}$ until $A_{600} 0.6$ then expression induced at $18^{\circ} \mathrm{C}$ by addition of $0.5 \mathrm{mM}$ isopropyl- $\beta$-D-thiogalactopyranoside (IPTG) overnight. Cell lysates in phosphate buffer (20 mM phosphate, $\mathrm{pH} 7.4,0.5 \mathrm{M} \mathrm{NaCl})$ were prepared using a French press. Proteins 
were purified using Ni-NTA His-Bind Resin (Novagen Madison, WI) and a step elution with phosphate buffer containing $300 \mathrm{mM}$ imidazol. Purified proteins were incubated with His-tagged Tev protease at $30^{\circ} \mathrm{C}$ for $30 \mathrm{~min}$ to remove the MBP (Maltose binding protein)-His tag. After the tags and Tev protease were removed using Ni chromatography, the purified J proteins were dialyzed against two changes of dialysis buffer $(20 \mathrm{mM}$ Tris- $\mathrm{HCl}, \mathrm{pH} 8,150 \mathrm{mM} \mathrm{NaCl}, 3 \mathrm{mM}$ dithiothreitol (DTT), $10 \%$ glycerol). Ssa $1_{\triangle E E V D}$ and variant J protein plasmids were constructed using the QuikChange Site-Directed mutagenesis kit from Stratagene (La Jolla, CA). Hsp104 (pMal His Tev Hsp104) was purified in a manner similar to that described for Sis1 and Sse1 (Smt3 (SUMO)-His 6 fusion protein) was purified in a manner similar to that described for Apj1 in Sahi et al [20].

Previously described yeast strains of the W303 genetic background were used: JJ1146 (ydj1::HIS3 sis $1:: L E U 2)$ [14] and WY26 (sis $1:: L E U 2)$ [25]. To partially rescue the severe $\Delta y d j 1$ growth defect JJ1146 was transformed with pRS317-ydj1-N134 [14], which includes the codons for the N-terminal 134 residues and promoter of $Y D J 1$, and thus encodes the entire J-domain and glycine-rich region. Strains WY26 and JJ1146 [pRS317-ydj1-N134] were transformed with YCp50 carrying wild type or mutant SIS1. Resulting TRP + colonies were grown on synthetic defined media containing 5-fluoroorotic acid (5-FOA) (Toronto Research Chemicals, Inc.) to counterselect for YCp50-SIS1 [14]. To test for dominance (Fig. 7c) cells were plated prior to counterselection with (5-FOA), and thus were expressing wild type Sis1 from YCp50 and either wild type or variant Sis1 from pRS314.

\section{Luciferase refolding assay}


Unless otherwise stated luciferase refolding assays were carried out as follows: Firefly luciferase (2.5 $\mu \mathrm{M}$, Sigma-Aldrich, St. Louis, $\mathrm{MO}$ ) was denatured for $30 \mathrm{~min}$ at $30^{\circ} \mathrm{C}$ in buffer A ( $25 \mathrm{mM}$ HEPES, $\mathrm{pH} 7.4,50 \mathrm{mM} \mathrm{KCl}, 5 \mathrm{mM} \mathrm{MgCl} 2$ ) containing DTT (5 mM) and urea (6M). The denatured luciferase was diluted, to give a final concentration of $30 \mathrm{nM}$, in buffer A containing 2 mM ATP, J protein (Sis1, 1.6 $\mu \mathrm{M}$; Xdj1, $1.6 \mu \mathrm{M}$; Ydj1, $3.2 \mu \mathrm{M})$, Ssa1 $(0.8 \mu \mathrm{M}$ when with Sis1 or Xdj1;1.6 $\mu \mathrm{M}$ when with Ydj1), Sse1 (0.2 $\mu \mathrm{M}$ with Sis1, $0.1 \mu \mathrm{M}$ with Xdj1 or Ydj1), and Hsp104 $(1 \mu \mathrm{M})$ and incubated at $30^{\circ} \mathrm{C}$ for $1 \mathrm{~h}$. The amount of J protein (Xdj1, Ydj1, and Sis1), Ssa1 and Sse1 were set at the concentrations indicated above based on optimization tests over a range of concentrations: J proteins from $0.8 \mu \mathrm{M}$ to $6.4 \mu \mathrm{M}$, Ssa1 from $0.4 \mu \mathrm{M}$ to $3.2 \mu \mathrm{M}$; Sse1 from $0.05 \mu \mathrm{M}$ to $0.4 \mu \mathrm{M}$. To measure refolding $1 \mu \mathrm{l}$ of the refolding mixture was mixed with 24 $\mu l$ of buffer A supplemented with DTT to $1 \mathrm{mM}$ and bovine serum albumin (BSA) at $0.1 \mathrm{mg} / \mathrm{ml}$. $50 \mu \mathrm{l}$ of luciferase assay system (Promega, Madison, WI) was added. Measurements were taken in a BioTek synergy2 plate-reader.

Refolding was also carried out after denaturation of luciferase by incubation in buffer $\mathrm{A}$ containing $6 \mathrm{M}$ guanidinium- $\mathrm{HCl}$, in which case folding was achieved in the absence of Hsp104 or Sse1. The refolding mixture contained J protein (Sis1, Xdj1 or Ydj1) and Ssa1 at $3.2 \mu \mathrm{M}$ and $0.8 \mu \mathrm{M}$, respectively.

\section{MDH refolding assay}

Porcine heart MDH $(5 \mathrm{mg} / \mathrm{ml}$, Roche, Nutley, $\mathrm{NJ}))$ was denatured at $48^{\circ} \mathrm{C}$ for $30 \mathrm{~min}$ in refolding buffer (25 mM HEPES, pH 7.4, $50 \mathrm{mM} \mathrm{KCl,} 5 \mathrm{mM} \mathrm{MgCl}_{2}, 2 \mathrm{mM} \mathrm{ATP}$ ). After denaturation, MDH was diluted, to a final concentration of $500 \mathrm{nM}$, in refolding buffer containing chaperones (J proteins (Sis1, $3.2 \mu \mathrm{M} ; \mathrm{Xdj} 1,1.6 \mu \mathrm{M} ; \mathrm{Ydj} 1,1.6 \mu \mathrm{M}$ ), Ssa1 (1.6 $\mu \mathrm{M}$ for Sis1; $0.8 \mu \mathrm{M}$ for Xdj1 
and Ydj1), Sse1 (0.2 $\mu \mathrm{M}$ for Sis1; $0.1 \mu \mathrm{M}$ for Xdj1 and Ydj1), Hsp104 (1 $\mu \mathrm{M}))$, ADP (0.1 mM), creatine kinase $(87.5 \mathrm{U} / \mathrm{ml})$, creatine phosphate $(25 \mathrm{mM})$, and incubated at $30^{\circ} \mathrm{C}$. As for luciferase refolding assays, the amount of J proteins (Xdj1, Ydj1, and Sis1), Ssa1 and Sse1 were set at the concentrations indicated above based on optimization tests over a range of concentrations: J proteins from $1.6 \mu \mathrm{M}$ to $6.4 \mu \mathrm{M}$; Ssa1 from $0.8 \mu \mathrm{M}$ to $3.2 \mu \mathrm{M}$; Sse1 from 0.05 $\mu \mathrm{M}$ to $0.4 \mu \mathrm{M}$. MDH activity (absorbance at $340 \mathrm{~nm}$ ) was determined in a BioTek synergy2 plate-reader using $\beta$-NADH $(0.28 \mathrm{mM})$ and oxaloacetate $(0.5 \mathrm{mM}))$ in $\mathrm{MDH}$ activity buffer $(150$ $\mathrm{mM}$ potassium phosphate, $\mathrm{pH}$ 7.5, $10 \mathrm{mM}$ DTT, BSA $(1 \mathrm{mg} / \mathrm{ml}))$.

\section{Protein-Protein interaction ELISA assays}

Ssa1 Hsp70 (70 $\mu \mathrm{l}$ of $0.4 \mu \mathrm{M})$ in $100 \mathrm{mM} \mathrm{NaHCO} 3, \mathrm{pH} 8.6$ was bound to each well of a high binding plate (Costar 3369, Corning, NY). Wells were washed with phosphate-buffer saline (PBS), blocked with $0.5 \%$ BSA in PBS, and subsequently washed with PBS containing $0.2 \%$ tween 20 (PBST). Increasing amounts of Sis1 wild type and variants in PBST containing 0.25\% BSA were added and then incubated at room temperature (RT) for $2 \mathrm{~h}$. After incubation, the wells were washed with PBST and 1:1000 dilution of Sis1 polyclonal antisera was applied and incubation continued for $1.5 \mathrm{~h}$ at RT. After extensive washing with PBST and subsequent addition of a 1:4000 dilution of donkey anti-rabbit $\mathrm{IgG}$, horseradish peroxidase linked whole antibody (GE Healthcare, Piscataway, NJ) was added, incubation was continued for $1 \mathrm{~h}$. The wells were then washed again using PBST and the reaction was developed by peroxidase substrate, ABTS (2, 2'-azino-bis (3-ethylbenzthiazoline-6-sulphonic acid)). Absorbance measurements were taken at $415 \mathrm{~nm}$. 


\section{Single-turnover ATPase assays}

Single-turnover ATPase assays of Ssal were performed as described in Sahi et al [20]. ${ }^{32} \mathrm{P}-\alpha-$ ATP-Ssal complexes were incubated with a 2 -fold molar excess of $\mathrm{J}$ proteins at $24^{\circ} \mathrm{C}$. Aliquots were applied to a TLC plate for detection of ATP and ADP. The amount of ATP hydrolyzed to ADP over time was determined using Imagequant TL (GE).

\section{Protein crystallization}

Several N-terminal fragments of Sis1 protein from S. cerevisiae, varying in length over the range of 67-127 residues, were designed to test protein expression and crystallization. The ORFs were amplified with KOD DNA polymerase using conditions and reagents provided by Novagen, Madison, WI. The DNA fragments were cloned into the pMCSG7 vector [33] using a modified LIC protocol [34]. This process generated expression clones producing a fusion protein with an N-terminal $\mathrm{His}_{6}$ affinity tag and a TEV protease recognition site. The proteins were expressed and purified using standard procedures on an AKTAxpress automated purification system (GE/Amersham) [35]. The concentration of purified protein was determined utilizing a ND-1000 spectro-photometer system (NanoDrop Technologies). The tag was removed as described above,except incubation with TEV protease was carried out for 49 hours at $4^{\circ} \mathrm{C}$. The purified proteins were dialyzed in crystallization buffer (20 mM HEPES pH 8.0, $250 \mathrm{mM} \mathrm{NaCl}, 2 \mathrm{mM}$ DTT) for 24 hours and concentrated using a Centricon Plus-20 concentrator with a MW cut off of 5,000 Da (Millipore Corp.).

Crystallization conditions were determined with a sparse crystallization matrix at $4^{\circ} \mathrm{C}$ and $16^{\circ} \mathrm{C}$ using the sitting-drop vapor-diffusion technique. The best crystals were obtained for the construct expressing 89-residue protein from $2.4 \mathrm{M}$ sodium malonate $\mathrm{pH} 7.0$ after 7 days of 
incubation at $16^{\circ} \mathrm{C}$. Crystals selected for data collection were soaked in the crystallization buffer supplemented with $28 \%$ sucrose and flash-cooled in liquid nitrogen.

\section{Data collection, structure determination and refinement}

Single-wavelength X-ray diffraction data were collected at $100 \mathrm{~K}$ temperature at the 19 -ID beamline of the Structural Biology Center [36] at the Advanced Photon Source at Argonne National Laboratory using the program SBCcollect. The intensities were integrated and scaled with the HKL3000 suite [37].

The structure was determined by molecular replacement using MOLREP program [38] from CCP4 suite using the J-domain of human DnaJ homolog subfamily B member 8 as a search model (PDB ID: 2DMX) [22]. This NMR structure was modified by manual truncation and was used as the starting model for model building and refinement. Several rounds of manual adjustment of structure models using COOT [39] and refinement with Refmac program [40] from CCP4 suite [22] were performed. The stereochemistry of the structure was validated with PHENIX suite [41] incorporating MOLPROBITY tools [42]. Atomic coordinates and structure factors were deposited into the Protein Data Bank as 4RWU.

\section{Acknowledgements}

We thank Jaroslaw Marszalek for insightful discussions, Minyi Gu for providing Sis1 clones and and all members of the Structural Biology Center at Argonne National Laboratory for their help in conducting X-ray diffraction data collection, which is operated by the University of Chicago Argonne, LLC, for the U.S. Department of Energy, Office of Biological and Environmental 
Research under contract DE-AC02-06CH11357. This work was supported by National Institutes of Health Grants GM31107 (EAC) and GM094585 (AJ). The work of M.B. was supported by Polish National Science Center Grant 2013/09/N/NZ2/01979.

\section{References}

[1] Saibil H. Chaperone machines for protein folding, unfolding and disaggregation. Nature reviews Molecular cell biology. 2013;14:630-42.

[2] Kim YE, Hipp MS, Bracher A, Hayer-Hartl M, Hartl FU. Molecular chaperone functions in protein folding and proteostasis. Annual review of biochemistry. 2013;82:323-55.

[3] Sontag EM, Vonk WI, Frydman J. Sorting out the trash: the spatial nature of eukaryotic protein quality control. Current opinion in cell biology. 2014;26:139-46.

[4] Mayer MP. Hsp70 chaperone dynamics and molecular mechanism. Trends in biochemical sciences. 2013;38:507-14.

[5] Kampinga HH, Craig EA. The HSP70 chaperone machinery: J proteins as drivers of functional specificity. Nature reviews Molecular cell biology. 2010;11:579-92.

[6] Freeman BC, Myers MP, Schumacher R, Morimoto RI. Identification of a regulatory motif in Hsp70 that affects ATPase activity, substrate binding and interaction with HDJ-1. The EMBO journal. 1995;14:2281-92.

[7] Young JC, Hoogenraad NJ, Hartl FU. Molecular chaperones Hsp90 and Hsp70 deliver preproteins to the mitochondrial import receptor Tom70. Cell. 2003;112:41-50.

[8] Scheufler C, Brinker A, Bourenkov G, Pegoraro S, Moroder L, Bartunik H, et al. Structure of TPR domain-peptide complexes: critical elements in the assembly of the Hsp70-Hsp90 multichaperone machine. Cell. 2000;101:199-210. 
[9] Wu Y, Sha B. Crystal structure of yeast mitochondrial outer membrane translocon member Tom70p. Nature structural \& molecular biology. 2006;13:589-93.

[10] Odunuga OO, Hornby JA, Bies C, Zimmermann R, Pugh DJ, Blatch GL. Tetratricopeptide repeat motif-mediated Hsc70-mSTI1 interaction. Molecular characterization of the critical contacts for successful binding and specificity. The Journal of biological chemistry. 2003;278:6896-904.

[11] Alvira S, Cuellar J, Rohl A, Yamamoto S, Itoh H, Alfonso C, et al. Structural characterization of the substrate transfer mechanism in Hsp70/Hsp90 folding machinery mediated by Hop. Nature communications. 2014;5:5484.

[12] Li J, Wu Y, Qian X, Sha B. Crystal structure of yeast Sis1 peptide-binding fragment and Hsp70 Ssa1 C-terminal complex. The Biochemical journal. 2006;398:353-60.

[13] Aron R, Lopez N, Walter W, Craig EA, Johnson J. In vivo bipartite interaction between the Hsp40 Sis1 and Hsp70 in Saccharomyces cerevisiae. Genetics. 2005;169:1873-82.

[14] Johnson JL, Craig EA. An essential role for the substrate-binding region of Hsp40s in Saccharomyces cerevisiae. The Journal of cell biology. 2001;152:851-6.

[15] Fan CY, Lee S, Ren HY, Cyr DM. Exchangeable chaperone modules contribute to specification of type I and type II Hsp40 cellular function. Molecular biology of the cell. 2004;15:761-73.

[16] Caplan AJ, Douglas MG. Characterization of YDJ1: a yeast homologue of the bacterial dnaJ protein. The Journal of cell biology. 1991;114:609-21.

[17] Park SH, Kukushkin Y, Gupta R, Chen T, Konagai A, Hipp MS, et al. PolyQ proteins interfere with nuclear degradation of cytosolic proteins by sequestering the Sis $1 \mathrm{p}$ chaperone. Cell. 2013;154:134-45. 
[18] Shiber A, Breuer W, Brandeis M, Ravid T. Ubiquitin conjugation triggers misfolded protein sequestration into quality control foci when Hsp70 chaperone levels are limiting. Molecular biology of the cell. 2013;24:2076-87.

[19] Summers DW, Wolfe KJ, Ren HY, Cyr DM. The Type II Hsp40 Sis1 cooperates with Hsp70 and the E3 ligase Ubr1 to promote degradation of terminally misfolded cytosolic protein. PloS one. 2013;8:e52099.

[20] Sahi C, Kominek J, Ziegelhoffer T, Yu HY, Baranowski M, Marszalek J, et al. Sequential duplications of an ancient member of the DnaJ-family expanded the functional chaperone network in the eukaryotic cytosol. Molecular biology and evolution. 2013;30:985-98. [21] Lu Z, Cyr DM. Protein folding activity of Hsp70 is modified differentially by the hsp40 cochaperones Sis1 and Ydj1. The Journal of biological chemistry. 1998;273:27824-30. [22] Szyperski T, Pellecchia M, Wall D, Georgopoulos C, Wuthrich K. NMR structure determination of the Escherichia coli DnaJ molecular chaperone: secondary structure and backbone fold of the $\mathrm{N}$-terminal region (residues 2-108) containing the highly conserved J domain. Proceedings of the National Academy of Sciences of the United States of America. 1994;91:11343-7.

[23] Luke MM, Sutton A, Arndt KT. Characterization of SIS1, a Saccharomyces cerevisiae homologue of bacterial dnaJ proteins. The Journal of cell biology. 1991;114:623-38.

[24] Aron R, Higurashi T, Sahi C, Craig EA. J-protein co-chaperone Sis1 required for generation of [RNQ+] seeds necessary for prion propagation. The EMBO journal. 2007;26:3794-803. [25] Yan W, Craig EA. The glycine-phenylalanine-rich region determines the specificity of the yeast Hsp40 Sis1. Molecular and cellular biology. 1999;19:7751-8. 
[26] Sondheimer N, Lopez N, Craig EA, Lindquist S. The role of Sis1 in the maintenance of the [RNQ+] prion. The EMBO journal. 2001;20:2435-42.

[27] Wang M, Weiss M, Simonovic M, Haertinger G, Schrimpf SP, Hengartner MO, et al.

PaxDb, a database of protein abundance averages across all three domains of life. Molecular $\&$ cellular proteomics : MCP. 2012;11:492-500.

[28] Malinovska L, Kroschwald S, Munder MC, Richter D, Alberti S. Molecular chaperones and stress-inducible protein-sorting factors coordinate the spatiotemporal distribution of protein aggregates. Molecular biology of the cell. 2012;23:3041-56.

[29] Alberti S. Molecular mechanisms of spatial protein quality control. Prion. 2012;6:437-42.

[30] Suzuki H, Noguchi S, Arakawa H, Tokida T, Hashimoto M, Satow Y. Peptide-binding sites as revealed by the crystal structures of the human Hsp40 Hdj1 C-terminal domain in complex with the octapeptide from human Hsp70. Biochemistry. 2010;49:8577-84.

[31] Pfund C, Huang P, Lopez-Hoyo N, Craig EA. Divergent functional properties of the ribosome-associated molecular chaperone Ssb compared with other Hsp70s. Molecular biology of the cell. $2001 ; 12: 3773-82$.

[32] Meyer AE, Hoover LA, Craig EA. The cytosolic J-protein, Jjj1, and Rei1 function in the removal of the pre-60 S subunit factor Arx1. The Journal of biological chemistry. 2010;285:9618.

[33] Stols L, Gu M, Dieckman L, Raffen R, Collart FR, Donnelly MI. A new vector for highthroughput, ligation-independent cloning encoding a tobacco etch virus protease cleavage site. Protein expression and purification. 2002;25:8-15.

[34] Dieckman L, Gu M, Stols L, Donnelly MI, Collart FR. High throughput methods for gene cloning and expression. Protein expression and purification. 2002;25:1-7. 
[35] Kim Y, Dementieva I, Zhou M, Wu R, Lezondra L, Quartey P, et al. Automation of protein purification for structural genomics. Journal of structural and functional genomics. 2004;5:111-8. [36] Rosenbaum G, Alkire RW, Evans G, Rotella FJ, Lazarski K, Zhang RG, et al. The Structural Biology Center 19ID undulator beamline: facility specifications and protein crystallographic results. Journal of synchrotron radiation. 2006;13:30-45.

[37] Minor W, Cymborowski M, Otwinowski Z, Chruszcz M. HKL-3000: the integration of data reduction and structure solution--from diffraction images to an initial model in minutes. Acta crystallographica Section D, Biological crystallography. 2006;62:859-66.

[38] Vagin A, Teplyakov A. Molecular replacement with MOLREP. Acta crystallographica Section D, Biological crystallography. 2010;66:22-5.

[39] Emsley P, Cowtan K. Coot: model-building tools for molecular graphics. Acta crystallographica Section D, Biological crystallography. 2004;60:2126-32.

[40] Murshudov GN, Vagin AA, Dodson EJ. Refinement of macromolecular structures by the maximum-likelihood method. Acta crystallographica Section D, Biological crystallography. 1997;53:240-55.

[41] Adams PD, Grosse-Kunstleve RW, Hung LW, Ioerger TR, McCoy AJ, Moriarty NW, et al. PHENIX: building new software for automated crystallographic structure determination. Acta crystallographica Section D, Biological crystallography. 2002;58:1948-54.

[42] Davis IW, Murray LW, Richardson JS, Richardson DC. MOLPROBITY: structure validation and all-atom contact analysis for nucleic acids and their complexes. Nucleic acids research. 2004;32:W615-9. 


\section{Figure Legends}

Fig. 1. EEVD Hsp70) important for Sis1, but not Ydj1/Xdj1, driven protein refolding. Luciferase or malate dehydrogenase (MDH) was denatured and diluted into various chaperone mixtures. Aliquots were removed at the indicated times and enzymatic activity determined. Activity of non-denatured enzyme was taken as $100 \%$. (a) Urea-denatured luciferase or (c) heatdenatured MDH was diluted into refolding buffer containing Sis1, Xdj1 or Ydj1 as the J-protein co-chaperone, in addition to Hsp104, Sse1 and either Ssa1 (Hsp70) or Ssa1 having EEVD deleted $\left(\mathrm{Hsp} 70_{\triangle \mathrm{EEVD}}\right)$. MDH activity at $90 \mathrm{~min}$ is shown; see Supplementary Fig. 1a for additional time points. (b) Luciferase treated with guanidinium hydrochloride was diluted into folding buffer containing only Sis1, Xdj1 or Ydj1 and Hsp70 or Hsp70 $0_{\triangle \mathrm{EEVD}}$. In (a) the two controls, Hsp70 without J-protein (black square) and Hsp70 $\triangle$ EEVD without J-protein (white square), overlap. Therefore, black squares are not visible.

\footnotetext{
Fig. 2. Substitution of $\mathrm{J}$ domain of Ydj1/Xdj1 for that of Sis1 overcomes Sis1 dependence on EEVD interaction. (a) Luciferase or (b) MDH was denatured by treatment with urea or heat, respectively, and diluted into chaperone-containing refolding buffer containing the indicated Jprotein co-chaperone and either Hsp70 or Hsp $70_{\triangle \mathrm{EEVD}}$, as indicated, in addition to Hsp104 and Sse1. MDH activity at 90 min is shown; see Supplementary Fig. 1b for additional time points. In (a) the two controls, Hsp70 without J-protein (black square) and Hsp70 $\triangle$ EEVD without Jprotein (white square), overlap. Therefore, black squares are not visible. (c) Isolated Hsp70-[ ${ }^{32} \mathrm{P}$ $\alpha$-ATP] complex was incubated with the indicated J-protein $(0.25 \mu \mathrm{M})$ and the release of ${ }^{32} \mathrm{P}$ determined with time.
} 
Fig. 3. Segment of helix 3 of J-domain is important for overcoming EEVD dependence.

(a) Alignment of J-domain of Xdj1, Ydj1 and Sis1, performed using DNASTAR (Madison, WI). Red lines (A,B, C) indicate regions of particular divergence and were changed in Sis1. Helices (1-4), based on homology with J-domain of Escherichia coli DnaJ (PDB ID: 1XBL) indicated.

(b) Luciferase or (c) MDH was denatured by treatment with urea or heat, respectively, and diluted into chaperone-containing refolding buffer containing wt Sis1 or variants having indicated alterations in Sis1 J-domain making it more similar to Ydj1, with either full-length

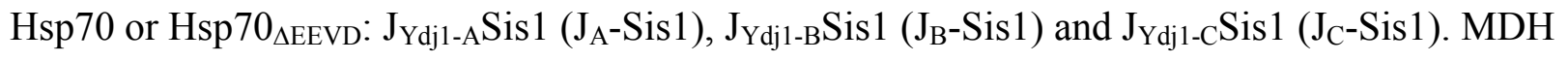
activity at $90 \mathrm{~min}$ is shown; see Supplementary Fig. 1c for additional time points.

\author{
Fig. 4. Sis1 residue $\mathbf{E 5 0}$ of Sis1 $\mathrm{J}$ domain is key for overcoming dependence on EEVD \\ binding. (a) Luciferase (a) or MDH (b) was denatured by treatment with urea or heat, \\ respectively, and diluted into chaperone-containing refolding buffer containing wt Sis 1 or \\ variants having indicated alterations in Sis $1 \mathrm{~J}$-domain making it more similar to Ydj1, with either \\ full-length Hsp70 or Hsp70 ${ }_{\triangle \mathrm{EEVD}}$. MDH activity at $90 \mathrm{~min}$ is shown; see Supplementary Fig. 1d \\ for additional time points. (c) Isolated Hsp70-[ ${ }^{32} \mathrm{P} \alpha$-ATP $]$ complex was incubated with the \\ indicated J-protein $(0.25 \mu \mathrm{M})$ and the release of ${ }^{32} \mathrm{P}$ determined with time.
}

\footnotetext{
Fig. 5 Sis1 intramolecular interaction between E50 of J domain and R73 of glycine-rich region. (a) Top: Line diagram of Sis1 domains, with residues at endpoints of domains indicated: J-domain (J); glycine-rich (G-rich), C-terminal domains (CTD) and dimerization domain (DD). Bottom-left: Crystal structure of $S$. cerevisiae Sis1 N-terminal 1-81 residue fragment (PDB ID: 4RWU), as ribbon representation generated using Pymol (DeLano Scientific LL). The side
} 
chains of E50 and R73 residues are shown as sticks. Bottom-right: Blow-up of the double saltbridge region formed by E50 (oxygen in red) and R73 (nitrogen in blue). Hydrogen bonds are shown as dashed lines with lengths as indicated. (PDB\#: 4RWU) (b, c). Luciferase (b) or MDH (c) was denatured by treatment with urea or heat, respectively, and diluted into chaperonecontaining refolding buffer containing either wt Sis1, no Sis1 (-) or Sis1variants having E50A or R73A substitutions, in addition to Hsp104, Sse1 and Ssa1. In (b) the two controls, Hsp70 without J-protein (black square) and Hsp70 $\triangle \mathrm{EEVD}$ without J-protein (white square), overlap. Therefore, black squares are not visible. MDH activity at $90 \mathrm{~min}$ is shown; see Supplementary Fig. 1e for additional time points.

Fig. 6 Analysis of Sis1 variants defective in EEVD interaction. (a) Left: Three-dimensional structure of Sis1 CTD1 (gray) in complex with extreme C-terminus of Ssa1 Hsp70 (red) (PDB: 2B26). Key lysines on Sis1 for Ssa1 interaction in blue. Right: interaction of indicated Sis1 variants with Hsp70 using ELISA assay. Ssa1 Hsp70 was bound in wells and incubated with increasing concentrations of Sis1 variants. After washing, bound Sis1 was detected using antiSis1 antibody. Maximum binding observed for wt Sis1 was set at 100\%. (b, c) Luciferase or MDH was denatured by treatment with urea or heat, respectively, and diluted into chaperonecontaining refolding buffer that included Hsp104 and Sse1. MDH activity at 90 min is shown; see Supplementary Fig. 1f, g for additional time points.

\author{
Fig 7. Sis1 variant defective in EEVD interaction suppresses growth defect caused by \\ reduced Ydj1 function. (a-c) 10-fold serial dilutions of the indicated strains were plated on \\ minimal medium and incubated at indicated temperatures. (a) $\Delta$ sis 1 expressing Sis 1 (WT) or
}




\author{
$\operatorname{Sis} 1_{\mathrm{K} 199 \mathrm{~N} / \mathrm{K} 202 \mathrm{~N} / \mathrm{K} 214 \mathrm{~N}}(\mathrm{~K} 199 / 202 / 204 \mathrm{~N}): 2$ days $\left(23^{\circ}, 30^{\circ}, 37^{\circ} \mathrm{C}\right)$. (b) ydj $1_{1-134} \Delta$ sis 1 expressing \\ either Sis1 (WT) or Sis1 $1_{\mathrm{K} 199 \mathrm{~N} / \mathrm{K} 202 \mathrm{~N} / \mathrm{K} 214 \mathrm{~N}}(\mathrm{~K} 199 / \mathrm{K} 202 / \mathrm{K} 204 \mathrm{~N}): 2$ days $\left(30^{\circ}, 34^{\circ} \mathrm{C}\right)$ or 3 days \\ $\left(23^{\circ} \mathrm{C}\right) .(\mathrm{c})$ as in (b) except cells contained an additional plasmid having a wild type copy of SIS1

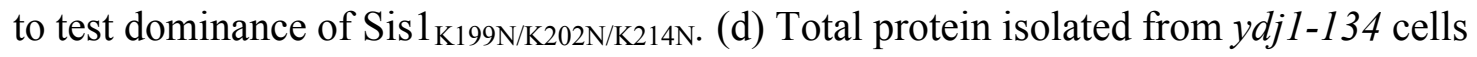 \\ expressing either Sis1 (WT) or Sis $1_{\mathrm{K} 199 \mathrm{~N}, \mathrm{~K} 202 \mathrm{~N}, \mathrm{~K} 214 \mathrm{~N}}(\mathrm{~K} 199,202,204 \mathrm{~N})$ was resolved by \\ electrophoresis, electroblotted to nitrocellulose, and probed with antibody to Sis1, and, as a \\ loading control, Ssa (control).
}

Fig 8. Model: EEVD(Hsp70) interaction with Sis1 and EEVD receptor of Hsp90 pathway. EEVD(Hsp70) binds both Sis1 and EEVD receptors, such as that of the Hsp90 system, but does not bind Ydj1. Thus, Ydj1 can bind, and efficiently transfer its client, to an Hsp70 already bound to an EEVD receptor (heavy solid arrow). However, since binding of EEVD(Hsp70) to Sis1 and to EEVD receptors is mutually exclusive, Sis 1-bound clients are more poorly transferred to Hsp70 prebound to EEVD receptors (thin dotted arrow) and thus into the Hsp90 pathway. The

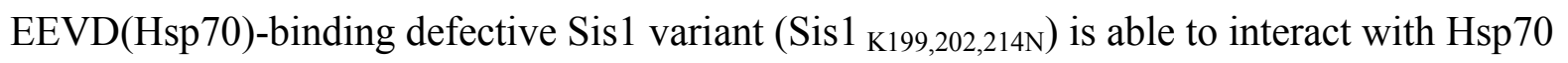
prebound to receptors without competition (heavy solid arrow), and thus able to compensate better than wild-type Sis 1 for disruption of Ydj1 function (see Fig 7). Not shown: EEVD receptors also exist on the mitochondrial outer membrane, acting in an analogous manner to Hsp90 EEVD binding adaptor proteins. 
Fig. 1.

(a)

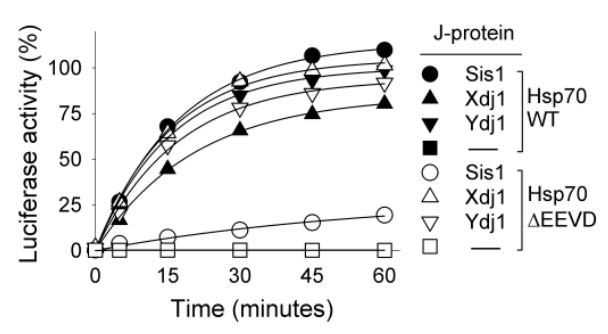

(c)

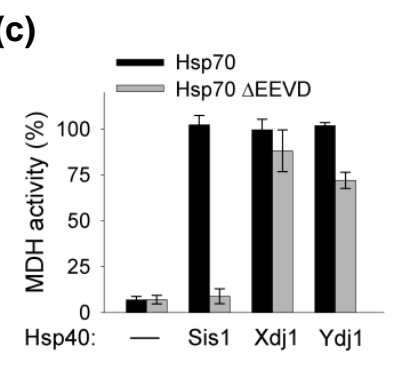

Fiqure

(b)

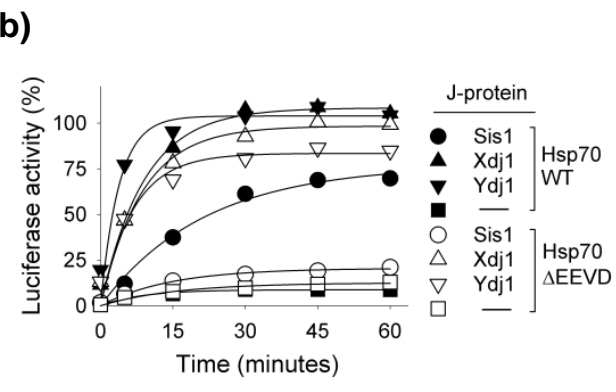


Fig. 2.

(a)

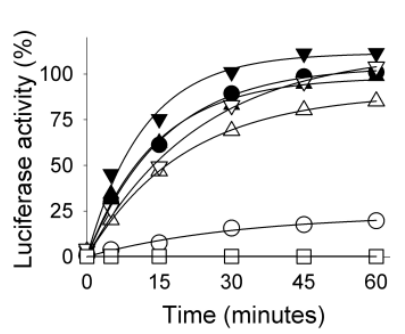

(b)

J-protein

- $\mathrm{J}_{\mathrm{Xdj} 1} \mathrm{Sis}$ Hsp70

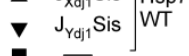

Sis1

$\triangle J_{\mathrm{Xdj} 1}$ Sis Hsp70

$\left.\nabla \quad \mathrm{J}_{\mathrm{Ydj} 1} \mathrm{Sis}\right] \Delta \mathrm{EEVD}$

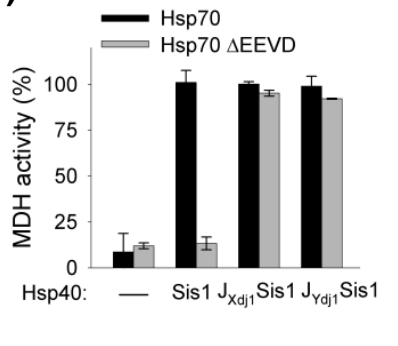

(c)

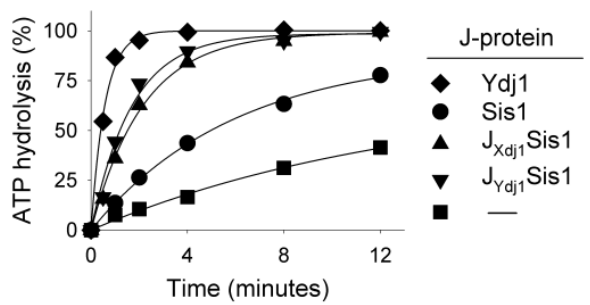


Fig. 3.

(a)
H1
H2
H3
$\mathrm{H} 4$

MSGSDRGDRL YDVLGVTRDATVDEI KTAYRKLALKHHPDKYVDQDSKEVNEI KFKEI TAAYEI LSDPEKKSHYDL

MSGSDRGDRL YDVLGVTRDATVQEI KTAYRKLALKHHPDKYVDQDSKEVNEI KFKEI TAAYEI LSDPEKKSHYDL

Xdj1 MSGSDRGDRLYDVLGVTRDATVQEI KTAYRKLALKHHPDKYVDQDSKEVNEIKFKEI TAAYEI LSDPEKKSHYDL 75

Ydj1 - - MVKETKFYDI LGVPVTATDVEIKKAYRKCALKYHPDKNPSEE- - - AAEKFKEASAAYEI LSDPEKRDI YDQ 68

Sis1 - - MVKETKLYDLLGVSPSANEQELKKGYRKAALKYHPDKPTG- - - DTEKFKEI SEAFEI LNDPQKRELYDQ 66

(b)

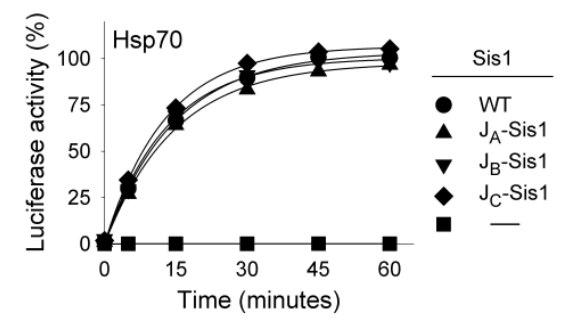

(c)

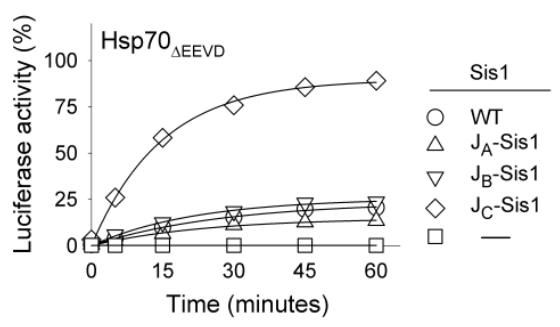

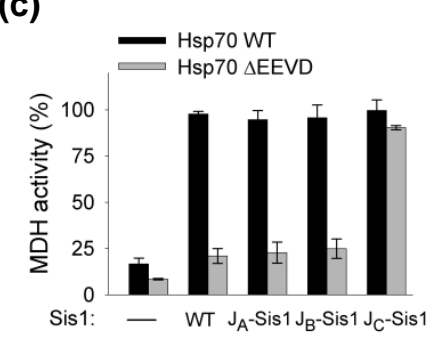



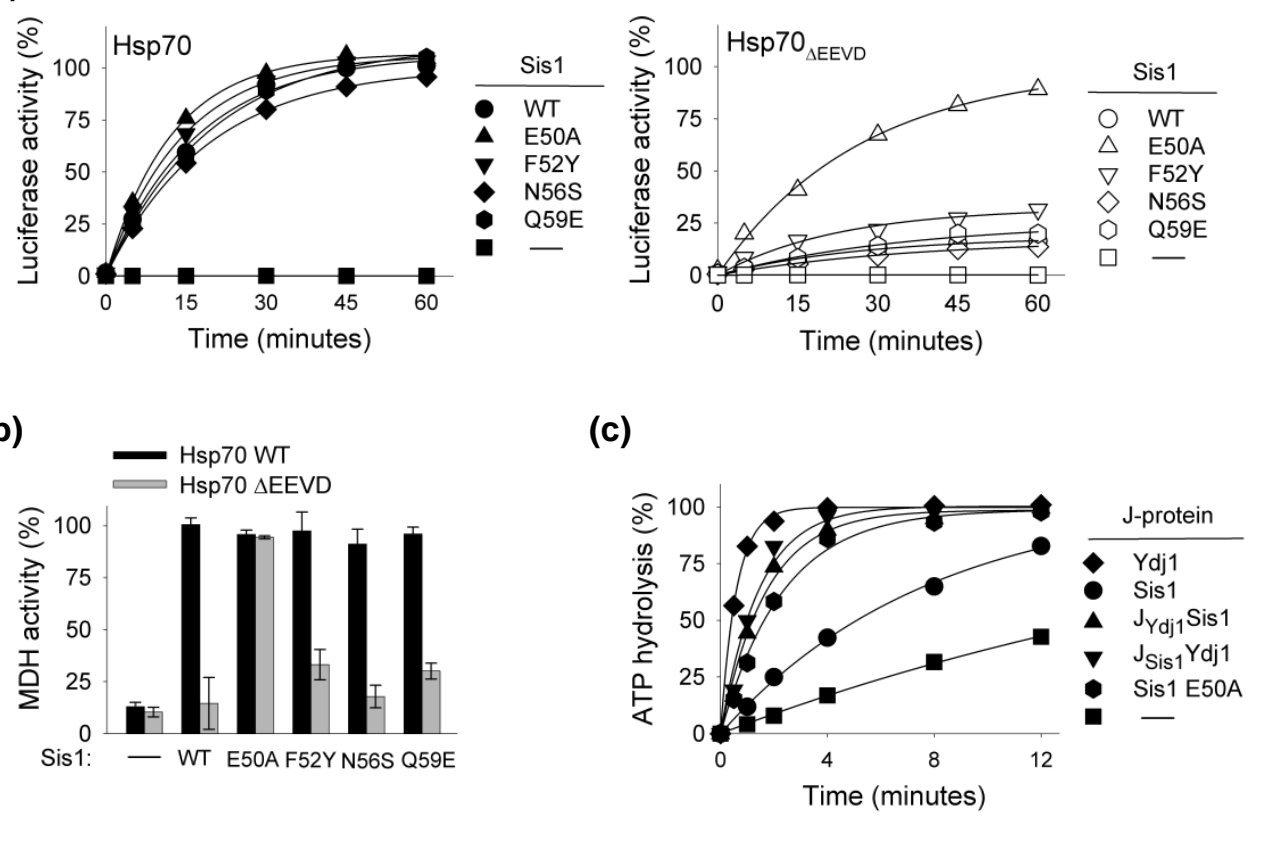

Figure

(a)

(b)

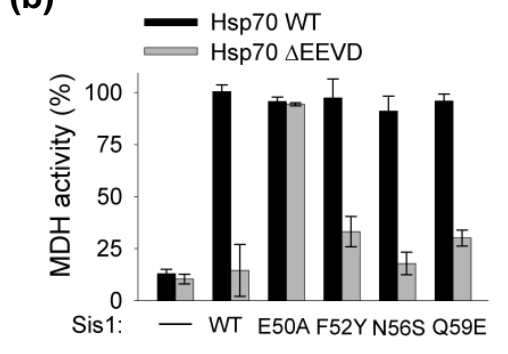

\section{Fig. 4. \\ Fig. 4.}

(c) 
Figure

Fig. 5.

(a)

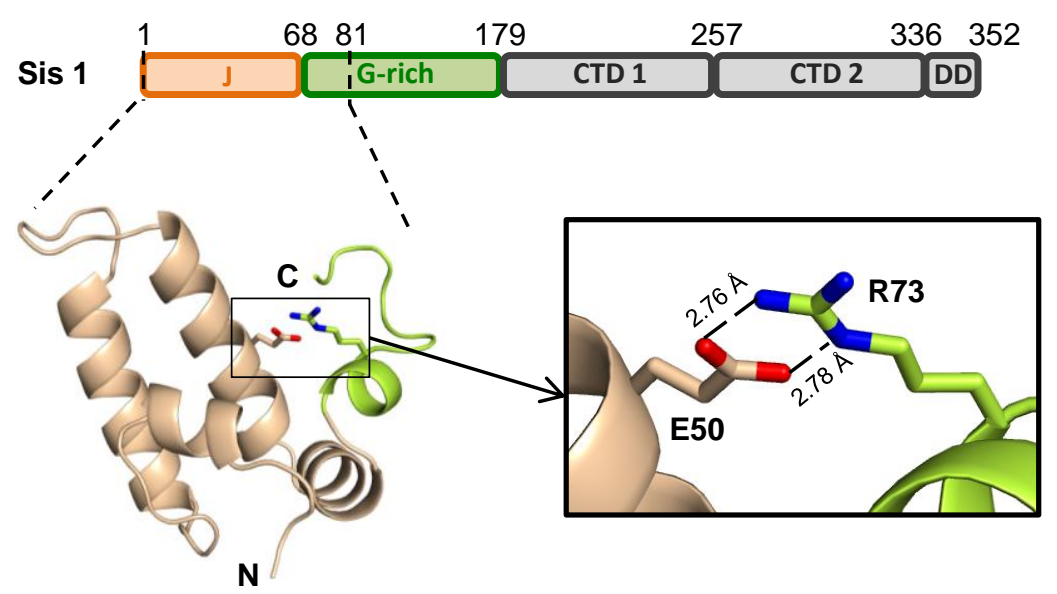

(b)

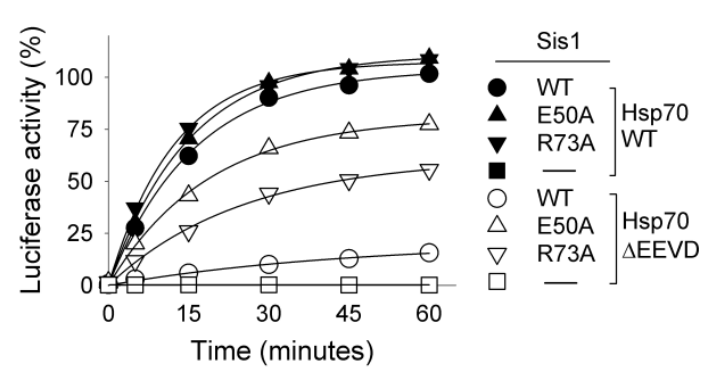

(c)

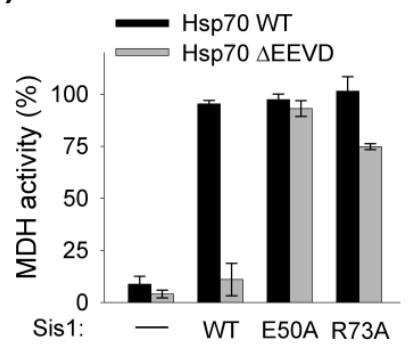



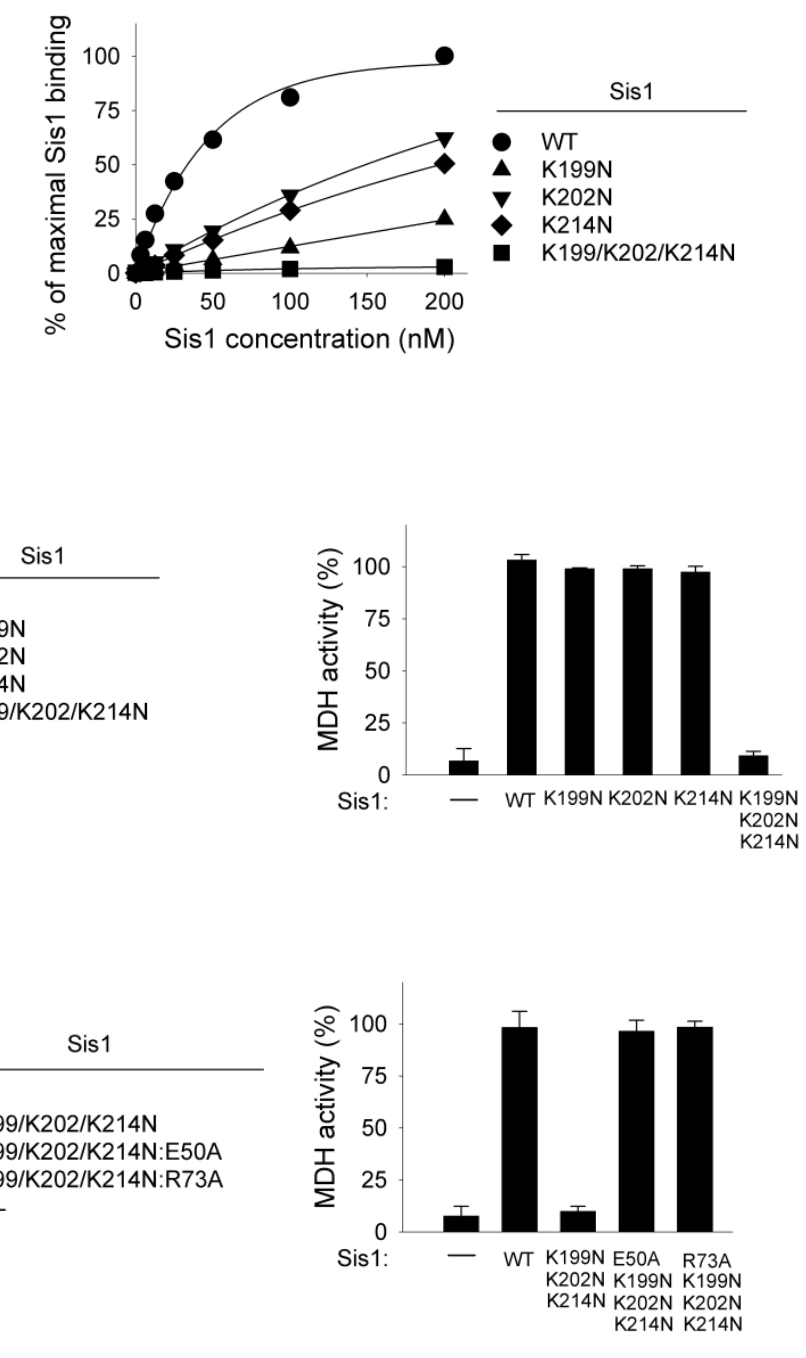

Figure

(a)

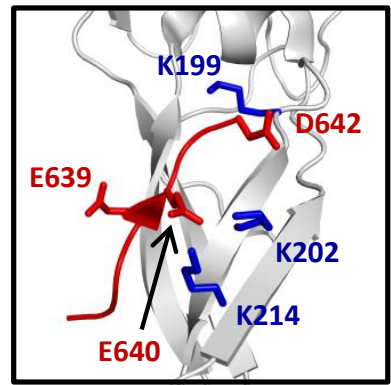

(b)

(c)

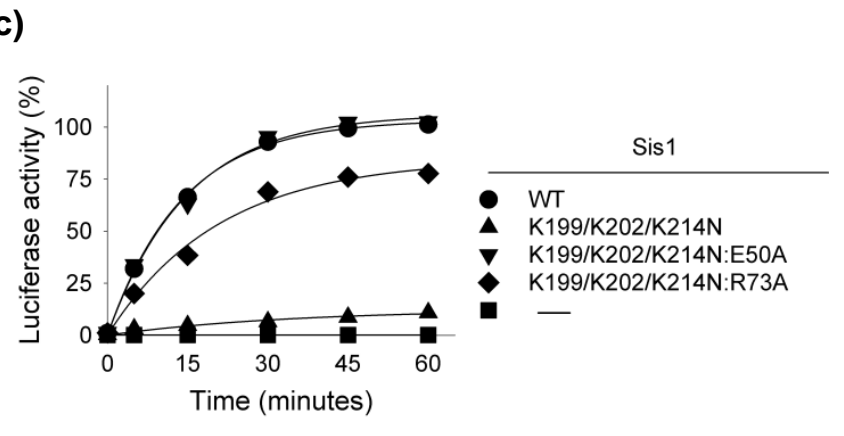

\section{Fig. 6.}

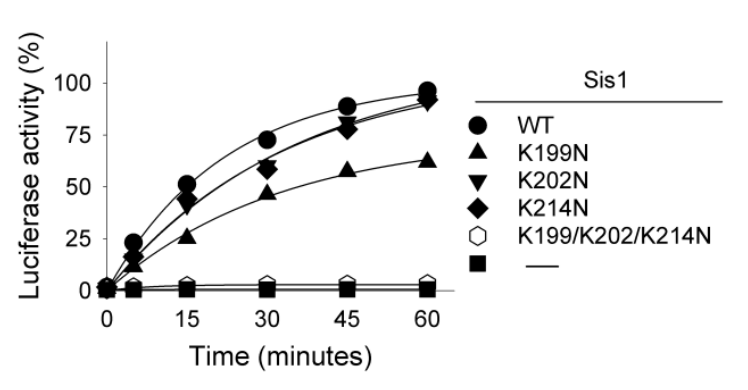

)

$$
\text { Time (minutes) }
$$


Figure

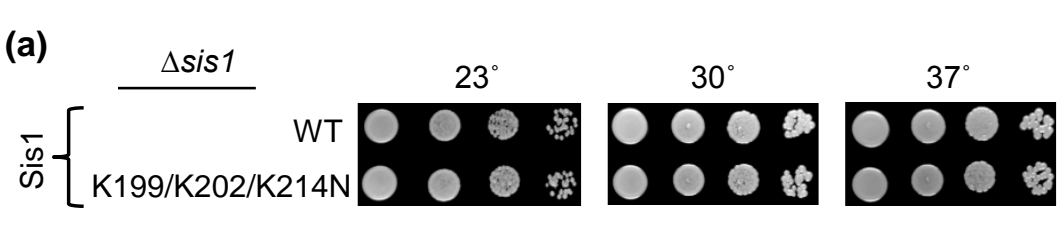

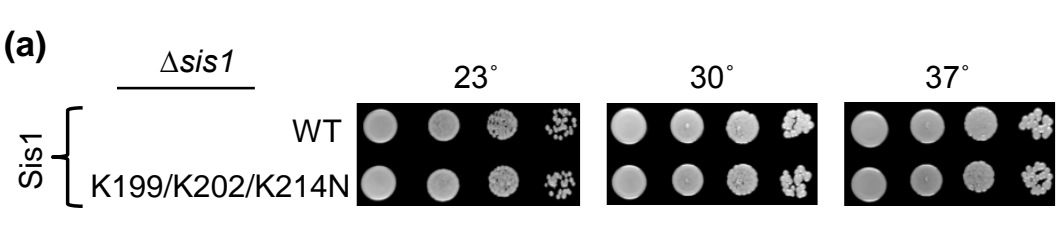

Fig. 7.

Figure
(b)

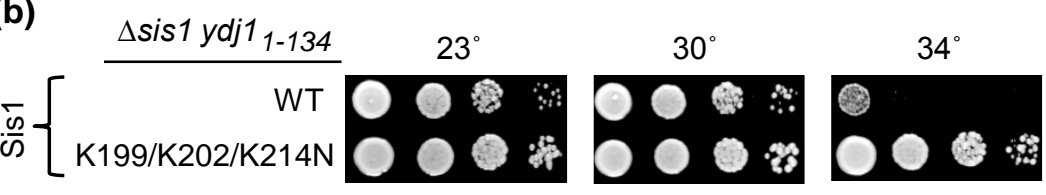

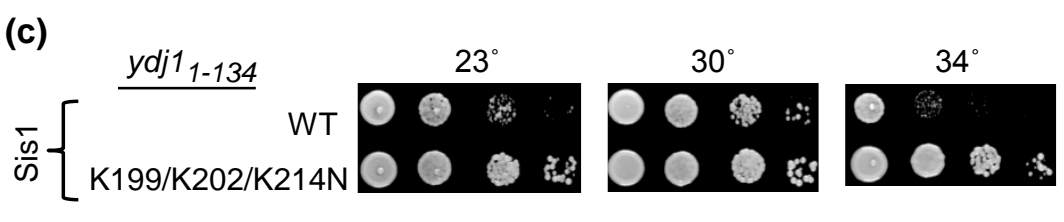

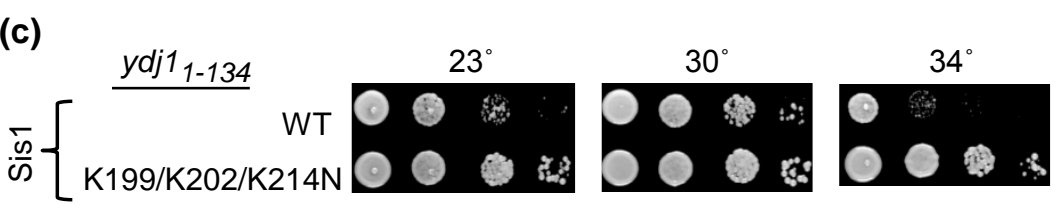

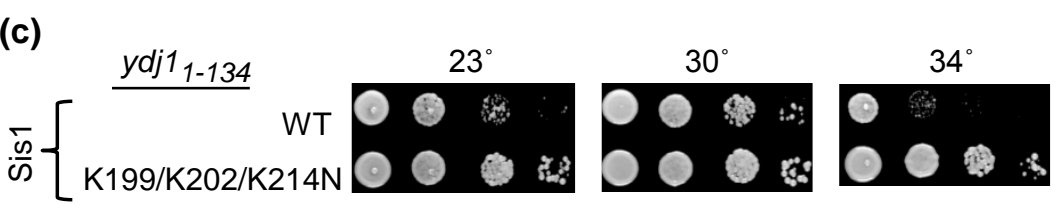

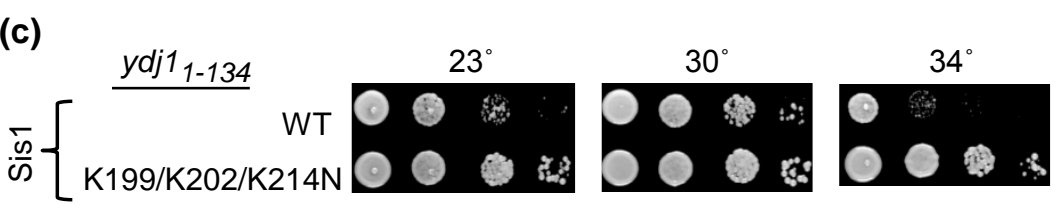
(d)

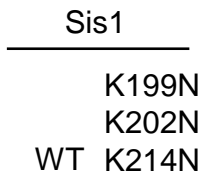
$\longrightarrow$ Sis1
- control

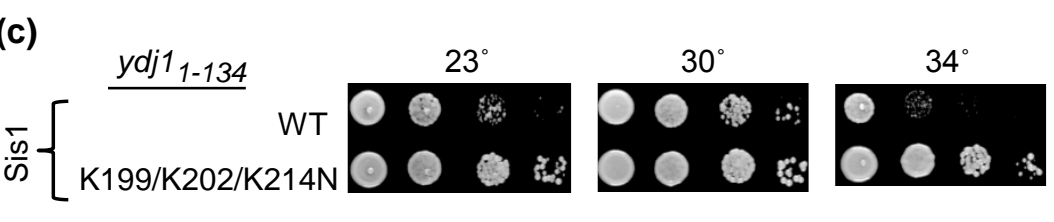

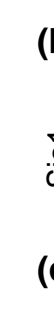


Fig.8.

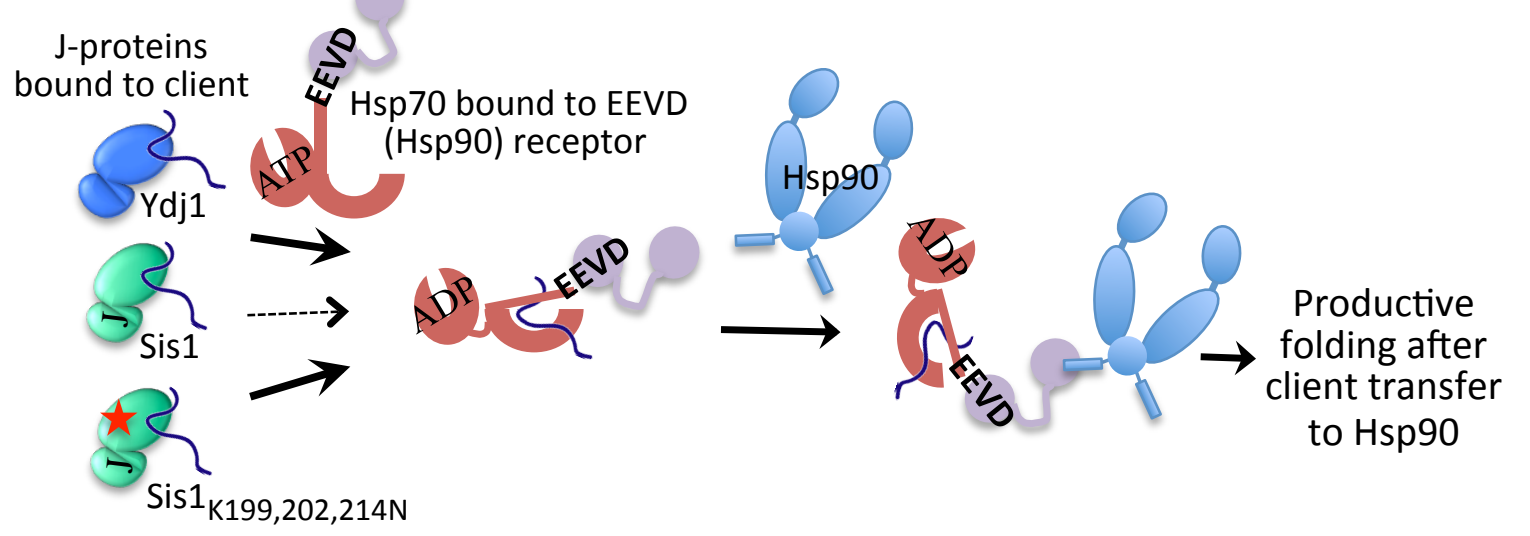




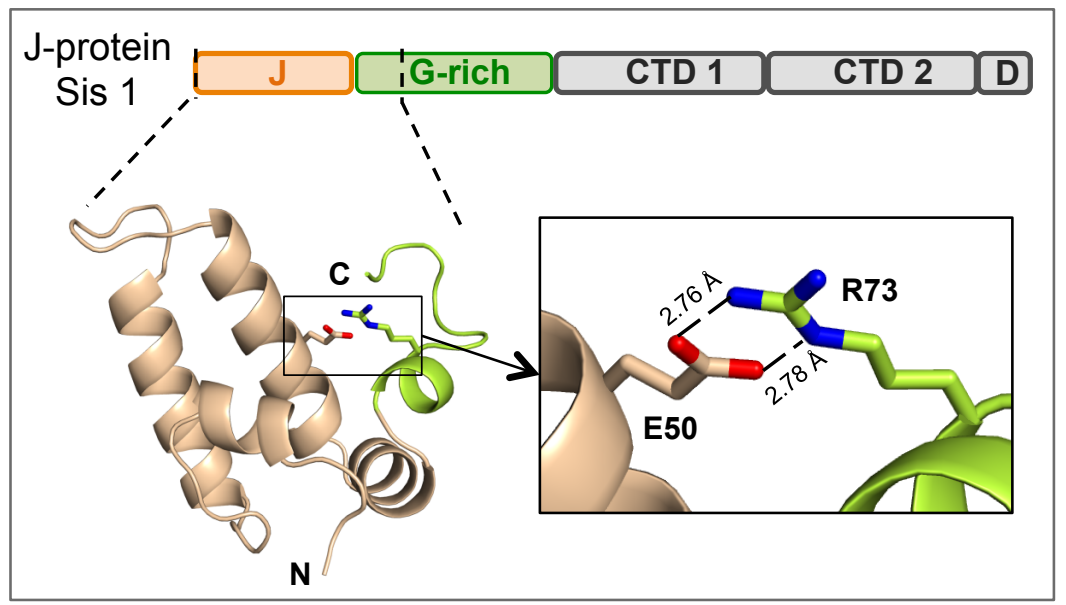

